\title{
Effect of Losartan and Amlodipine on Heart Rate Variability In Essential Hypertensive Patients
}

\author{
Shamima Sultana ${ }^{1}$, Shelina Begum ${ }^{2}$, Sultana Ferdousi ${ }^{3}$, Mohammad Nesar Uddin Ahmed ${ }^{4}$
}

\begin{abstract}
Background: Essential hypertension is characterized by sympathovagal imbalance that is responsible for arrhythmias and sudden cardiac death. Antihypertensive drugs restores sympatho vagal balance. Losartan and amlodipine are common antihypertensive drugs. Objective: To compare the effect of losartan and amlodipine on heart rate variability (HRV) in hypertensive patients . Methods: This prospective analytical study was carried out in the Department of Physiology, Bangabandhu Sheikh Mujib Medical University (BSMMU), Shahbag, Dhaka from July 2012 to June 2013. For this study, 120 newly diagnosed hypertensive patients without any treatment (group B, age 30-55 years) were selected from the Out Patients Department of cardiology, BSMMU, Dhaka on their first day of visit. 60 apparently healthy normotensive subjects were also studied as control (group A). HRV of the patients were measured both before and after they were treated with two different antihypertensive drugs (losartan and amlodipine) separately. According to the selection of drug these patients were divided into two groups. Group B1 included 60 patients received losartan $50 \mathrm{mg}$ daily and group B2 included 60 patients treated by amlodipine $5 \mathrm{mg}$ daily. They were observed once before the treatment $\left(\mathrm{B} 1_{\mathrm{a}} \& \mathrm{~B} 2_{\mathrm{a}}\right)$ and after 3 months medication $\left(\mathrm{B} 1_{\mathrm{b}} \& \mathrm{~B} 2_{\mathrm{b}}\right)$ and also after 6 months medication $\left(\mathrm{B} 1_{\mathrm{c}} \& \mathrm{~B} 2_{\mathrm{c}}\right.$ ). For assessing HRV, Mean heart rate (HR), Mean R-R interval, Max/Min R-R interval, SDNN, RMSSD were recorded by a polyrite. Data were compared among before treatment, after 3 months treatment and after 6 months treatment. For statistical analysis ANOVA, independent sample ' $t$ ' test and paired sample ' $t$ ' were performed. Results: Mean resting pulse rate, mean heart rate, systolic blood pressure (SBP), diastolic blood pressure (DBP) were significantly higher $(\mathrm{P}<0.001)$ and mean R-R interval, SDNN, RMSSD were significantly lower $(\mathrm{P}<0.001)$ in patients before treatment compared to those of healthy normotensive subjects and to the values after treatment. In both drug groups, SDNN, RMSSD, mean R-R interval were found significantly higher after 6 months of treatment compared to their values after 3 months treatment and also close to the values in normotensive subjects. In losartan group RMSSD were found significantly higher $(\mathrm{p}<0.01)$ and mean R-R interval,SDNN were found though higher but not significant than the corresponding values in amlodipine treated patients after 6 months treatment. Conclusion: Vagal modulation is decreased in untreated hypertensive patients which is increased by treatment with both losartan and amlodipine but the effect is more pronounced in losartan. The decreased autonomic function was improved better with longer treatment duration.
\end{abstract}

Key words: Newly diagnosed hypertensive patients, heart rate variability,losartan, amlodipine.

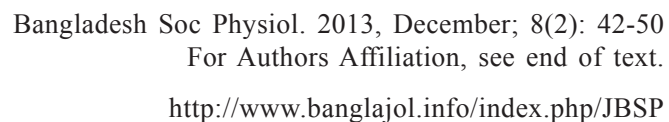

Received April 2013; $\quad$ Accepted November 2093

J Bangladesh Soc Physiol. 2013, December; 8(2): 42-50 


\section{Introduction}

Hypertension is usually defined as persistent blood pressure at 140/90 $\mathrm{mm} \mathrm{Hg}$ or higher, affects about a quarter of the adult population in many countries. ${ }^{1}$ The autonomic nervous system (ANS) plays an important role in the control of arterial blood pressure and heart rate and intimately related to the development of systemic hypertension. 1

Essential hypertension is associated with altered autonomic nerve function which is characterized by sympathetic overactivity, attenuation of parasympathetic modulation of the heart and overall reduced heart rate variability $(\mathrm{HRV}){ }^{2-6}$

Several studies have identified reduced HRV in essential hypertension. ${ }^{3,7-9}$ Reduced HRV has been associated with a higher risk for all mortality in survivors of an acute myocardial infarction 10-13 and sudden cardiac death. ${ }^{14}$ The antihypertensive drug therapy reportedly can reduce the risk of stroke and coronary heart disease 34\% and $21 \%$, respectively ${ }^{15,16}$. Effect of some antihypertensive drugs individually or in combinations on HRV has been studied in hypertensive patients. ${ }^{17-20}$

The heart rate variability is a reliable indicator of the cardiac autonomic nerve function. ${ }^{5}$ Among the various HRV measures mean R-R interval, mean heart rate, maximum and minimum $\mathrm{R}-\mathrm{R}$ ratio, SDNN and RMSSD usually used as marker for cardiac vagal activity. ${ }^{21}$

It has been reported that various antihypertensive drugs modify the sympathoparasympathetic balance. ${ }^{3,22}$ Literature review present conflicting reports on the effect of losartan and amlodipine on cardiac autonomic activity. Few studies reported losartan inhibit sympathetic activity in hypertensive patients but others found no significant effect ${ }^{23-25}$. Similar reports were also found about the effect of amlodipine. ${ }^{26-30}$
In addition to the conflicting result of this two drugs, no study so far compared their effect on HRV. Therefore this study aimed to observe and compare the effect of losartan and amlodipine on HRV in hypertension as well as the time period of the therapy required to achieve their target.

\section{Methods}

This prospective analytical study was carried out to observe the cardiac autonomic activity by assessing some time domain measures of HRV in 120 adult male hypertensive patients $(<159 / 99)^{31}$ aged 30-55 years in the Department of Physiology, Bangabandhu Sheikh Mujib Medical University (BSMMU), Shahbag, Dhaka from July 2012to June 2013. For comparison 60 apparently healthy normotensive subjects $(<139 / 89)^{31}$ were also studied as control (group A). According to drug and time period of treatment, study subjects were divided into two groups(B1 and B2). GroupB1 included 60 patients received losartan $50 \mathrm{mg}$ daily and $\mathrm{B} 2$ included 60 patients received amlodipine $5 \mathrm{mg}$ daily. They were observed once before the treatment $\left(\mathrm{B} 1_{\mathrm{a}} \& \mathrm{~B} 2_{\mathrm{a}}\right)$ after3 months medication $\left(B 1_{b} \& B 2_{b}\right)$ and after 6 months medication $\left(B 1_{c}\right.$ $\left.\& B 2_{c}\right)$. These patients were selected from the Out Patient Department of Cardiology, BSMMU. Hypertensive patients with history of taking antihypertensive drugs, previously diagnosed as hypertension, diabetes mellitus, ischaemic heart diseases, renal disease, psychic disorder and smoking were excluded from the study. After selection, the objectives of the study were explained to all the subjects and their voluntary participation was encouraged. An informed written consent was taken from each subject. The protocol of this study was approved by the Institutional Review Board of BSMMU. Then the subject was prepared for Autonomic Nerve Function Test. Immediately after enrollment, HRV data were recorded before initiating treatment 
with any antihypertensive drugs. All the autonomic nerve function tests were done in the Autonomic Nerve Function laboratory in the department of Physiology. The subject was kept in supine position in a bed for 15-20 minutes in a controlled laboratory environment. Then all preparations for recording of the Heart rate variability parameters were made by connecting the channels of ECG and a 5 minutes short term recording was taken in resting position. Mean heart rate, Mean R-R interval, Max/Min R-R interval, SDNN, RMSSD were measured by a polygraph.

Then all the patients were requested to attend the Department of Physiology of BSMMU, after 3 months and after 6 months of antihypertensive medication for recording of follow up HRV data. For statistical analysis ANOVA, independent sample ' $t$ ' test and paired sample ' $t$ ' were performed.

\section{Results}

Anthopometric data of all subjects are given in Table-I.

Mean resting pulse, SBP and DBP were significantly higher in group $\mathrm{B}_{1 \mathrm{a}}$ and $\mathrm{B}_{2 \mathrm{a}}$ compared to those of controls and this values were significantly decreased in group $\mathrm{B}_{1 \mathrm{~b}}, \mathrm{~B}_{1 \mathrm{c}}$, $\mathrm{B}_{2 \mathrm{~b}}$ and $\mathrm{B}_{2 \mathrm{c}}$ compared to those before treatment. (Table II).

Mean values of mean HR were significantly higher $(\mathrm{p}<.001)$ in group $\mathrm{B}_{1 \mathrm{a}}$ and $\mathrm{B}_{2 \mathrm{a}}$ and Mean R-R interval, Max/Min R-R, SDNN, RMSSD were significantly lower $(\mathrm{p}<0.001)$ in group $\mathrm{B}_{1 \mathrm{a}}$ and $\mathrm{B}_{2 \mathrm{a}}$ than those of group A. Mean heart rate were significantly decreased $(p<0.001)$ in group $B_{1 b}$, $\mathrm{B}_{1 \mathrm{c},}, \mathrm{B}_{2 \mathrm{~b}}$ and group $\mathrm{B}_{2 \mathrm{c}}$ in comparison to their baseline values. In addition this value showed no significant difference when compared between group $A$ and group $B_{1 c}$. Mean heart rate in group $B_{2 c}$ was significantly higher $(p<0.05)$ than that of group $B_{1 c}$. Mean values of Mean R-R interval, Max/Min R-R interval, SDNN, RMSSD were significantly increased in group $B_{1 b}, B_{1 c}$, $\mathrm{B}_{2 \mathrm{~b}}$ and $\mathrm{B}_{2 \mathrm{c}}$ when compared to those of baseline values. But mean values of SDNN and RMSSD were significantly decreased in group $B_{1 c}$ and group $\mathrm{B}_{2 \mathrm{c}}$ compared to those of control group. RMSSD in group $\mathrm{B}_{1 \mathrm{c}}$ was found significantly higher $(\mathrm{p}<0.003)$ than that of group $2 \mathrm{C}$ (Table III \& IV).

Table I : Anthopometric data of all subjects $(\mathrm{n}=120)$

\begin{tabular}{|c|c|c|}
\hline Group & Age (years) & $\operatorname{BMI}\left(\mathrm{kg} / \mathrm{m}^{2}\right)$ \\
\hline \multirow[t]{2}{*}{$A(n=60)$} & $42.04 \pm 1.134$ & $23.24 \pm 0.243$ \\
\hline & $(30-35)$ & $(18.59-24.77)$ \\
\hline \multirow[t]{2}{*}{$\mathrm{B}_{1 \mathrm{a}}(\mathrm{n}=60)$} & $41.64 \pm 1.165$ & $23.27 \pm 0.216$ \\
\hline & $(30-35)$ & $(18.65-24.77)$ \\
\hline \multirow[t]{2}{*}{$\mathrm{B}_{2 \mathrm{a}}(\mathrm{n}=60)$} & $42.74 \pm 1.312$ & $23.36 \pm 0.198$ \\
\hline & $(30-35)$ & $(18.37-24.74)$ \\
\hline \multicolumn{3}{|c|}{ Statistical analysis } \\
\hline$A$ vs $B_{1 a}$ vs $B_{2 a}^{\hat{i}}$ & $0.296^{\mathrm{ns}}$ & $0.850^{\mathrm{ns}}$ \\
\hline$A$ vs $B_{1 a}{ }^{o ̈}$ & $0.806^{\mathrm{ns}}$ & $0.912^{\mathrm{ns}}$ \\
\hline$A$ vs $B_{2 a}{ }^{\ddot{o}}$ & $0.688^{\mathrm{ns}}$ & $0.688^{\mathrm{ns}}$ \\
\hline $\mathrm{B}_{1 \mathrm{a}} \mathrm{vs} \mathrm{B}_{2 \mathrm{a}}{ }^{\mathrm{U}}$ & $0.533^{\text {ns }}$ & $0.748^{\mathrm{ns}}$ \\
\hline
\end{tabular}

Data were expressed as mean $\pm \mathrm{SE}$, figures in parenthesis indicate ranges.. $\ddot{o}=$ independent sample ' $t$ ' test. U'=paired sample ' $\mathrm{t}$ ' test.

$\mathrm{SBP}=$ Systolic blood pressure.

$\mathrm{DBP}=$ maximum

Group $\mathrm{A}=$ Apparently healthy subject (control)

B = Newly diagnosed hypertensive patients before treatment

$\mathrm{B}_{1 \mathrm{a}}=$ After 3 months treatment with losartan

$\mathrm{B}_{2 \mathrm{a}}=$ After 3 months treatment with amlodipine

$* * *=\mathrm{p}<0.001 \mathrm{~ns}=$ non significant $(\mathrm{p}>0.05)$

$* *=\mathrm{p}<0.01 \quad \mathrm{n}=$ number of subjects.

$*=p<0.05$ 
Table II : Baseline measures in different groups $(\mathrm{n}=120)$

\begin{tabular}{|c|c|c|c|}
\hline Groups & Pulse & Systolic blood pressure & Diastolic blood pressure \\
\hline \multirow[t]{2}{*}{ A } & $78.15 \pm 0.862$ & $126.4 \pm 0.603$ & $73.2 \pm 0.515$ \\
\hline & $(93-70)$ & $(120-135)$ & $(65-80)$ \\
\hline \multirow{2}{*}{$\mathrm{B}_{1 \mathrm{a}}$} & $83.55 \pm 0.919$ & $141.6 \pm 0.462$ & $91.5 \pm 0.295$ \\
\hline & $(95-70)$ & $(140-155)$ & (90-98) \\
\hline \multirow[t]{2}{*}{$\mathrm{B}_{1 \mathrm{~b}}$} & $76.37 \pm 0.767$ & $127.7 \pm 0.45$ & $77.4 \pm 0.510$ \\
\hline & $(90-70)$ & $(120-135)$ & $(70-85)$ \\
\hline \multirow[t]{2}{*}{$\mathrm{B}_{1 \mathrm{C}}$} & $75.33 \pm 0.664$ & $127.7 \pm 0.449$ & $76.2 \pm 0.476$ \\
\hline & $(87-65)$ & $(120-135)$ & $(70-85)$ \\
\hline \multirow[t]{2}{*}{$\mathrm{B}_{2 \mathrm{a}}$} & $82.33 \pm 0.994$ & $142.8 \pm 0.520$ & $91.2 \pm 0.265$ \\
\hline & $(95-70)$ & $(140-155)$ & $(90-95)$ \\
\hline \multirow[t]{2}{*}{$\mathrm{B}_{2 \mathrm{~b}}$} & $76.95 \pm 0.978$ & $128.2 \pm 0.474$ & $77.4 \pm 0.545$ \\
\hline & $(90-65)$ & $(120-135)$ & $(70-85)$ \\
\hline \multirow[t]{2}{*}{$\mathrm{B}_{2 \mathrm{c}}$} & $75.10 \pm 0.950$ & $127.4 \pm 0.381$ & $76.4 \pm 0.429$ \\
\hline & $(85-63)$ & $(120-130)$ & $(70-80)$ \\
\hline \multicolumn{4}{|l|}{ Statistical analysis } \\
\hline \multirow{2}{*}{$\begin{array}{c}A \operatorname{vs~} B_{1 a} \text { vs } B_{2 a} \text { vs } B_{3 a}{ }^{\hat{1}} \\
A \text { vs } B_{1 a} \ddot{o}\end{array}$} & $0.000 * * *$ & $0.000 * * *$ & $0.000 * * *$ \\
\hline & $0.000 * * *$ & $0.000 * * *$ & $0.000^{* * *}$ \\
\hline$A$ vs $B_{2 a}{ }^{o ̈}$ & $0.002^{* *}$ & $0.000 * * *$ & $0.000 * * *$ \\
\hline $\mathrm{B}_{1 \mathrm{a}} \mathrm{vs} \mathrm{B}_{1 \mathrm{~b}} \mathrm{U}$ & $0.000 * * *$ & $0.000 * * *$ & $0.000 * * *$ \\
\hline $\mathrm{B}_{1 \mathrm{~b}} \mathrm{vs} \mathrm{B}_{1 \mathrm{c}} \mathrm{U}$ & $0.252 \mathrm{~ns}$ & $0.095 \mathrm{~ns}$ & $0.047 *$ \\
\hline $\mathrm{B}_{1 \mathrm{a}} \mathrm{vs} \mathrm{B}_{1 \mathrm{c}}^{\mathrm{U}}$ & $0.000 * * *$ & $0.000 * * *$ & $0.000 * * *$ \\
\hline $\mathrm{B}_{2 \mathrm{a}}$ vs $\mathrm{B}_{2 \mathrm{~b}} \mathrm{U}$ & $0.000 * * *$ & $0.000^{* * *}$ & $0.000 * * *$ \\
\hline $\mathrm{B}_{2 \mathrm{~b}} \mathrm{vs} \mathrm{B}_{2 \mathrm{c}} \mathrm{U}$ & $0.105 \mathrm{~ns}$ & $0.090 \mathrm{~ns}$ & $0.051 \mathrm{~ns}$ \\
\hline $\mathrm{B}_{2 \mathrm{a}}$ vs B $\mathrm{B}_{2 \mathrm{c}}^{\mathrm{U}}$ & $0.000 * * *$ & $0.000 * * *$ & $0.000^{* * *}$ \\
\hline
\end{tabular}

Data were expressed as mean $\pm \mathrm{SE}$, figures in parenthesis indicate ranges.. $\mathrm{o}=$ independent sample ' $\mathrm{t}$ ' test. Ù=paired sample ' $t$ ' test.

$\mathrm{SBP}=$ Systolic blood pressure.

DBP $=$ Diastolic blood pressure

Group $\mathrm{A}=$ Apparently healthy subject (control)

$\mathrm{B}_{1 \mathrm{a}} \quad=$ Newly diagnosed hypertensive patients before treatment

$\mathrm{B}_{1 \mathrm{~b}}=$ After 3 months treatment with losartan

$\mathrm{B}_{1 \mathrm{c}} \quad=$ After 3 months treatment with losartan

$\mathrm{B}_{2 \mathrm{a}} \quad=$ Newly diagnosed hypertensive patients before treatment

$\mathrm{B}_{2 \mathrm{~b}} \quad=$ After 3 months treatment with amlodipine

$\mathrm{B}_{2 \mathrm{c}} \quad=$ After 3 months treatment with amlodipine

$* * * \quad=\mathrm{p}<0.001$

ns $\quad=$ non significant $(\mathrm{p}>0.05)$

$* * \quad=\mathrm{p}<0.01 \mathrm{n}=$ number of subjects.

$* \quad=\mathrm{p}<0.05$ 
Table III : Simple time domain measures of HRV in different groups $(\mathrm{n}=120)$

\begin{tabular}{|c|c|c|c|}
\hline Groups & $\begin{array}{c}\text { Mean heart rate } \\
\text { (Beat/min) }\end{array}$ & $\begin{array}{c}\text { Mean R-R } \\
\text { interval (sec.) }\end{array}$ & $\begin{array}{c}\text { Max/min R-R } \\
\text { interval }\end{array}$ \\
\hline A & $73.33 \pm 0.712$ & $0.753 \pm 0.12$ & $1.75 \pm 0.066$ \\
\hline$(n=60)$ & $(85-65)$ & $(0.91-0.6)$ & (3.17-1.19) \\
\hline $\begin{array}{l}\mathrm{B}_{1 \mathrm{a}} \\
(\mathrm{n}=60)\end{array}$ & $\begin{array}{c}84.85 \pm 0.895 \\
(95-70)\end{array}$ & $\begin{array}{l}0.693 \pm 0.014 \\
(1.04-0.571)\end{array}$ & $\begin{array}{l}1.46 \pm 0.041 \\
(3.79-1.12)\end{array}$ \\
\hline $\mathrm{B}_{1 \mathrm{~b}}$ & $\begin{array}{c}77.75 \pm 0.909 \\
(90-65)\end{array}$ & $\begin{array}{c}0.730 \pm 0.10 \\
(1.128-0.619)\end{array}$ & $\begin{array}{c}1.71 \pm 0.64 \\
(4.99-1.104)\end{array}$ \\
\hline $\mathrm{B}_{1 \mathrm{c}}$ & $\begin{array}{c}74 \pm 0.886 \\
(85-63)\end{array}$ & $\begin{array}{l}0.748 \pm 0.011 \\
(1.135-.551)\end{array}$ & $\begin{array}{c}1.78 \pm 0.057 \\
(12.18-.789)\end{array}$ \\
\hline $\mathrm{B}_{2 \mathrm{a}}$ & $\begin{array}{c}82.33 \pm 0.242 \\
\quad(99-60)\end{array}$ & $\begin{array}{l}0.699 \pm 0.010 \\
(1.075-.602)\end{array}$ & $\begin{array}{l}1.58 \pm 0.071 \\
(3.39-1.12)\end{array}$ \\
\hline $\mathrm{B}_{2 \mathrm{~b}}$ & $\begin{array}{c}78.92 \pm 1.057 \\
(95-60)\end{array}$ & $\begin{array}{c}0.737 \pm 0.008 \\
(.996-.617)\end{array}$ & $\begin{array}{l}1.70 \pm 0.051 \\
(2.80-1.12)\end{array}$ \\
\hline $\mathrm{B}_{2 \mathrm{c}}$ & $\begin{array}{c}77.13 \pm 0.013 \\
(97-64)\end{array}$ & $\begin{array}{c}0.747 \pm 0.013 \\
(1.128-0.562)\end{array}$ & $\begin{array}{c}1.72 \pm 0.052 \\
(3.37-.37)\end{array}$ \\
\hline \multicolumn{4}{|c|}{ Statistical analysis } \\
\hline$A$ vs $B_{1 a}{ }^{\ddot{o}}$ & $0.000 * * *$ & $0.001 * * *$ & $0.000 * * *$ \\
\hline$A$ vs $B_{1 b} \ddot{~}$ & $0.000 * * *$ & $0.122 \mathrm{~ns}$ & $0.714 \mathrm{~ns}$ \\
\hline$A$ vs $B_{1 c}{ }^{1 b}$ & $0.243 \mathrm{~ns}$ & $0.747 \mathrm{~ns}$ & $0.694 n s$ \\
\hline $\mathrm{B}_{1 \mathrm{a}}$ vs $\mathrm{B}_{1 \mathrm{~b}}$ U & $0.000 * * *$ & $0.024^{*}$ & $0.002 * *$ \\
\hline $\mathrm{B}_{1 \mathrm{~b}}$ vs B $\mathrm{B}_{1 \mathrm{c}} \mathrm{U}$ & $0.008^{* *}$ & 0.239 & $0.411 \mathrm{~ns}$ \\
\hline $\mathrm{B}_{1 \mathrm{a}}$ vs $\mathrm{B}_{1 \mathrm{c}} \mathrm{c}$ & $0.000^{* * *}$ & $0.004^{* *}$ & $0.000^{* *}$ \\
\hline $\mathrm{A} \mathrm{vs} \mathrm{B}_{2 \mathrm{a}} \stackrel{\mathrm{lc}}{\mathrm{or}}$ & $0.000 * * *$ & $0.001 * *$ & $0.098 \mathrm{~ns}$ \\
\hline $\mathrm{A}$ vs $\mathrm{B}_{2 \mathrm{~b}} \ddot{\mathrm{o}}$ & $0.000 * * *$ & $0.219 \mathrm{~ns}$ & $0.617 \mathrm{~ns}$ \\
\hline $\mathrm{A}$ vs B $\mathrm{B}_{2 \mathrm{c}} \mathrm{b}_{\ddot{\mathrm{o}}}$ & $0.003^{* *}$ & $0.742 \mathrm{~ns}$ & $0.746 \mathrm{~ns}$ \\
\hline $\mathrm{B}_{2}$ vs B $\mathrm{B}_{2 \mathrm{~b}} \mathrm{U}$ & $0.004^{* *}$ & $0.006^{* *}$ & $0.161 \mathrm{~ns}$ \\
\hline $\mathrm{B}_{2 \mathrm{~b}}$ vs B $\mathrm{B}_{2 \mathrm{c}} \mathrm{U}$ & $0.164 \mathrm{~ns}$ & $0.431 \mathrm{~ns}$ & $0.827 \mathrm{~ns}$ \\
\hline $\mathrm{B}_{2 \mathrm{a}}$ vs B $\mathrm{B}_{2 \mathrm{c}} \mathrm{U}$ & $0.000^{* * *}$ & $0.002 * *$ & $0.115 \mathrm{~ns}$ \\
\hline
\end{tabular}

Data were expressed as mean $\pm \mathrm{SE}$, figures in parenthesis indicate ranges. $\ddot{O}=$ independent sample ' $\mathrm{t}$ ' test. Ù=paired sample ' $t$ ' test.

$\mathrm{R}-\mathrm{R}=$ interval between successive $\mathrm{QRS}$ complex(sec)

Max $=$ maximum, Min $=$ minimum

Group A = Apparently healthy subject (control)

$\mathrm{B}_{1 \mathrm{a}} \quad=$ Newly diagnosed hypertensive patients before treatment

$\mathrm{B}_{1 \mathrm{~b}} \quad=$ After 3 months treatment with losartan

$\mathrm{B}_{1 \mathrm{c}} \quad=$ After 3 months treatment with losartan

$\mathrm{B}_{2 \mathrm{a}} \quad=$ Newly diagnosed hypertensive patients before treatment

$\mathrm{B}_{2 \mathrm{~b}} \quad=$ After 3 months treatment with amlodipine

$\mathrm{B}_{2 \mathrm{c}} \quad=$ After 3 months treatment with amlodipine

$* * * \quad=\mathrm{p}<0.001$

ns $\quad=$ non significant $(\mathrm{p}>0.05)$

$* * \quad=\mathrm{p}<0.01 \mathrm{n}=$ number of subjects.

* $\quad=\mathrm{p}<0.05$

J Bangladesh Soc Physiol. 2013, December; 8(2): 42-50 
Table IV: Statistical time domain measures of HRV in different groups $(\mathrm{n}=120)$

\begin{tabular}{|c|c|c|}
\hline Groups & SDNN (ms) & RMSSD (ms) \\
\hline \multirow[t]{2}{*}{$A(n=60)$} & $78.18 \pm 1.95$ & $32.69 \pm 0.89$ \\
\hline & $(99.8-50.09)$ & $(41.89-17.99)$ \\
\hline \multirow{2}{*}{$\mathrm{B}_{1 \mathrm{a}}(\mathrm{n}=60)$} & $40.63 \pm 2.37$ & $24.57 \pm 0.521$ \\
\hline & $(102.8-15.11)$ & $(32.99-18.76)$ \\
\hline \multirow{2}{*}{$\mathrm{B}_{1 \mathrm{~b}}$} & $54.40 \pm 2.39$ & $26.98 \pm 0.392$ \\
\hline & $(100.09-26.15)$ & $(34 .-22)$ \\
\hline \multirow{2}{*}{$\mathrm{B}_{1 \mathrm{c}}$} & $71.42 \pm 2.53$ & $30.59 \pm .414$ \\
\hline & $(108.1-31.09)$ & $(45.31-23.8)$ \\
\hline \multirow{2}{*}{$\mathrm{B}_{2 \mathrm{a}}$} & $50.97 \pm 1.94$ & $24.36 \pm .603$ \\
\hline & $(90.8-28.8)$ & $(34.99-15.78)$ \\
\hline \multirow{2}{*}{$\mathrm{B}_{2 \mathrm{~b}}$} & $52.4 \pm 2.50$ & $27.41 \pm .582$ \\
\hline & $(90.15-30.34)$ & $(36.77-20.6)$ \\
\hline \multirow{2}{*}{$\mathrm{B}_{2 \mathrm{c}}$} & $69.48 \pm 2.67$ & $28.56 \pm .528$ \\
\hline & $(100.9-40.88)$ & $(35.78-22.6)$ \\
\hline \multicolumn{3}{|c|}{ Statistical analysis } \\
\hline A vs $B_{1 a}{ }^{\ddot{o}}$ & $0.000 * * *$ & $0.000 * * *$ \\
\hline$A$ vs $B_{1 b} \ddot{0}$ & $0.000 * * *$ & $0.000 * * *$ \\
\hline$A$ vs $B_{1 c} \ddot{o}$ & 0.036 & 0.035 \\
\hline $\mathrm{B}_{1 \mathrm{a}}$ vs $\mathrm{B}_{1 \mathrm{~b}}$ Ù & $0.000 * * *$ & 0.001 \\
\hline $\mathrm{B}_{1 \mathrm{~b}}$ vs $\mathrm{B}_{1 \mathrm{c}} \mathrm{U}$ & $0.000 * * *$ & 0.008 \\
\hline $\mathrm{B}_{1 \mathrm{a}}$ vs B $_{1 \mathrm{c}} \mathrm{U}$ & $0.000 * *$ & $0.000 * * *$ \\
\hline $\mathrm{A}$ vs $\mathrm{B}_{2 \mathrm{a}}{ }^{\mathrm{o}}$ & $0.000 * * *$ & $0.000 * * *$ \\
\hline$A$ vs $B_{2 b} \ddot{o}$ & $0.000 * * *$ & $0.000 * * *$ \\
\hline$A$ vs $B_{2 c} \ddot{o}$ & $0.010^{*}$ & $0.000 * * *$ \\
\hline $\mathrm{B}_{2 \mathrm{a}}$ vs B $\mathrm{B}_{2 \mathrm{~b}}$. & $0.524 \mathrm{~ns}$ & $0.004 * *$ \\
\hline $\mathrm{B}_{2 b}$ vs B $\mathrm{B}_{2 \mathrm{c}} \mathrm{U}$ & $0.000 * * *$ & $0.076 \mathrm{~ns}$ \\
\hline $\mathrm{B}_{2 \mathrm{a}}$ vs B ${ }_{2 \mathrm{c}}^{\mathrm{U}}$ & $0.000 * * *$ & $0.000 * * *$ \\
\hline $\mathrm{B}_{1 \mathrm{c}} \operatorname{vs~B_{2c}}{ }^{\circ}$ & $0.586 \mathrm{~ns}$ & $0.003 * *$ \\
\hline
\end{tabular}

Data were expressed as mean $\pm \mathrm{SE}$, figures in parenthesis indicate ranges. $\ddot{o}=$ independent sample ' $t$ ' test. $\mathrm{U}=$ paired sample ' $t$ ' test.

SDNN $=$ Standard deviation of N-N interval

RMSSD $=$ Square root of mean squared differenceso between adjacent $\mathrm{NN}$ intervals.

Group

$\mathrm{A}=$ Apparently healthy subject (control)

$\mathrm{B}_{1 \mathrm{a}}=$ Newly diagnosed hypertensive patients before treatment

$\mathrm{B}_{1 \mathrm{~b}}=$ After 3 months treatment with losartan

$\mathrm{B}_{1 \mathrm{c}}=$ After 3 months treatment with losartan

$\mathrm{B}_{2 \mathrm{a}}=$ Newly diagnosed hypertensive patients before treatment

$\mathrm{B}_{2 \mathrm{~b}}=$ After 3 months treatment with amlodipine

$\mathrm{B}_{2 \mathrm{c}}=$ After 3 months treatment with amlodipine

$* * *=\mathrm{p}<0.001 \mathrm{~ns}=$ non significant $(\mathrm{p}>0.05)$

$* *=\mathrm{p}<0.01 \mathrm{n}=$ number of subjects. $*=\mathrm{p}<0.05$

\section{Discussion}

In this study effects of two antihypertensive drugs losartan and amlodipine on HRV in patients with essential hypertension were studied. HRVwas found reduced in hypertensive patients before initiating treatment with losartan and amlodipine compared to normotensive subjects which agrees to other investigators. ${ }^{18,20,26,32}$ The effect of losartan and amlodipine on HRV was observed after 3 months of medication and then again after 6 months of medication. It was observed that HRV was significantly increased after 3 months in losartan groups but though increased but not significant in amlodipine treated patients. For losartan, similar observation was made by Carpia et al ${ }^{25}$ and Chern et al. ${ }^{20}$ whereas others did not find this effect. For amlodipine, results of this study were almost similar to other investigators but they studied this effect with less duration of treatment. $27,31,32$

After 6 months of treatmen with these two drugs both losartan and amlodipine, HRV were found significantly increased compared to their corresponding values after their 3 months of treatment. Chern et al. ${ }^{20}$ observed similar effect of losartan after 6 months but they did not find any significant difference compared to 3 months treatment duration. No publications are available to compare this effect for amlodipine after 6 months treatment. The values of HRV after 6 months of treatment in both drugs were found close to the values of controls. Similar investigations found relative improvement of HRV after few weeks of treatment with both the drugs but it was still remained lower than the controls. ${ }^{25-27}$

In this study, time domain result reflct lower cardiac vagal modulation in untreated patients which was found significantly improved with both drugs after 3 and 6 months treatment.On further analysis the effect of losartan was found more pronounced than amlodipine improving the cardiac vagal activity which is supported by significantly higher value of RMSSD and lower 
value of mean HR in losartan treated patients. In addition, 6 months duration of treatment was found more effective than 3 months treatment which is supported by the facts that HRV values were close to controls after 6 months treatment.

Various mechanisms has been proposed for the improvement of HRV in hypertensive patients after treatment with losartan and amlodipine. Hypertension has been found associated with altered cardiac autonomic function evidenced by reduced HRV, sympathetic overactivity and low Baroreflex sensitivity (BRS). ${ }^{27,28,33}$

Losartan acts as Angiotensin II type-I receptor $\left(\mathrm{AT}_{1}\right)$ blocker which antagonizes the suppresor effect of elevated Angiotensin II on BRS thereby restore the sympathovagal by increasing BRS. Furthermore, it increases NO production from vascular endothelium and neuron which facilitate the regulation of BRS and HRV in losartan treated hypertensive patients. ${ }^{33,34}$

Amlodipine is a calcium channel antagonist, which inhibits norepinephrine release from the noradrenergic nerve terminals by blocking its calcium influx thus reducing sympathetic overactivity. This drug also suppresses Aldosterone secretion from adrenal glomerulosa cortex. ${ }^{35}$

Though both the drugs lower blood pressure by counter acting excess neural activity or BRS and also by suppressing adrenocortical hormone but their exact mechanisms for improvement of HRV after treatment with losartan and amlodipine in hypertensive groups can not be elucidated from this type of study. Moreover, the cause of the difference of their effect on cardiac autonomic function can not be anticipated from this study. However, the mentioned mechanisms for these two antihypertensive drug may have some influence on the improvement of cadiac autonomic activity in these patients after treatment with losatan and amlodipine. The outcome of the study suggests a positive relation and duration of treatment of these two antihypertenstve drugs with improvement of HRV.

\section{Conclusion}

Impairment of cardiac autonomic nervous activity occurred in untreated hypertensive patients which was improved by both losartan and amlodipine and in particular losartan was found more effective. The improvement of impaired cardiac autonomic function is better with longer duration of treatment.

\section{Author affiliations}

1. *Shamima Sultana, Medical Officer, Department of Physiology, Bangabandhu Sheikh Mujib Medical University (BSMMU), Bangladesh. Email: Sultanashamima68@yahoo.com

2. Shelina Begum, Professor, Chairman, Department of Physiology, Bangabandhu Sheikh Mujib Medical University (BSMMU), Bangladesh. Email: shelina1982@gmail.com

3. Sultana Ferdousi, Associate Professor, Department of Physiology, Bangabandhu Sheikh Mujib Medical University (BSMMU), Bangladesh. Email: sferdousiratna@gmail.com

4. Mohammad Nesar Uddin Ahmed

*For correspondence

\section{References}

1. Kjeldsen SE, Aksnes TA, Fagard RH and Mancia G. Hypertension. In: Cam A J, Luscher T F and Serruys P W (eds). The ESC of Cardiovascular Medicine. New York: Oxford University Press; 2009. P 437-464.

2. Julius S. Autonomic nervous system dysregulation in human hypertension. Am J Cardiol 1991; 67 : 3B-7B.

3. Guzzetti S, Piccaluga E, Casati R. Sympathetic predominance in essential hypertension: A study employing spectral analysis of heart rate variability. J Hypertens 1988 ; 6: 711-717.

4. Langewitz W, Ruddel H, Schachinger, H.F. Reduced parasympathetic cardiac control in patients with hypertension at rest and under mental stress. Am. Heart J 1994; 127:122-8.

5. Singh JP, Larson MG, Tsuji H. Reduced heart rate variability in new onset hypertension. Insights into pathogenesis of hypertension: The Framingham heart study. Hypertension 1998; 32:293-297.

6. Tabassum R, Begum N, Ferdousi S, Begum S, Ali T. Heart rate variability in patients with Essential 
Hypertension. J Bangladesh Soc Physiol 2010;5(1) : $1-7$.

7. Chakko S, Mulingtapang RF, Huikuri HV, Kessler KM, Materson BJ, Myeberg RJ. Alteration in heart rate variability and its circadian rhythm in hypertensive patients with left ventricular hypertrophy free of coronary artery disease. Am Heart J 1993;126 : 1364-1372.

8. Takalo R, Korhonen I, Turjanamaa V, Majahalme S, Tuomisto M, Uusitalo A. Short term variability of blood pressure and heart rate in borderline and mild hypersensitive subjects. Hypertension 1994; 23:18-24.

19. Huikuri HV, Ylitalo A, Pikkujamasa SM. Heart rate variability in systemic hypertension. Am J Cardiol 1996;77:1073-1077.

10. Kleiger RE, Miller JP, Bigger JT Jr, et al. Decreased heart rate variability and its association with increased mortality after acute myocardial infarction. Am J cardiol 1987; 59 : 256-262.

11. Vaishnav S. Stevenson R, Marchant B, Lagi K, Ranjadayalan K, Timmis A. Relation between heart rate variability early after acute myocardial infarctions and long term mortality. Am J Cardiol 1994 73: 653-657.

12. Lombardi F, Sandrone G, Pernpruder S, Sala R, Garimoldi M, Cerutti S, Baselli G, Pgani M, Malliani A. Heart rate variability as an index of sympathovagal interaction after acute myocardial infarction. Am J Cardiol 1987; 60:1239-1245.

13. Malik M, Farrell T, Camm AJ, Circadian rhythm of heart rate varibility after acute myocardial infarction and its influence on the prognostic value of heart rate variability. Am J Cardiol 1990; 66:1049-1054.

14. Martin GJ, Magid NM, Myers G, Barnett PS, Schaad JW, Weiss JS, Lesch M, Singer DH. Heart rate variability and sudden death secondary to coronary artery disease during ambulatory electrocardiographic monitoring. Am J Cardiol $1987 ; 60: 86-89$

15. Law M, Wald N, Morris J. Lowering blood pressure to prevent myocardial infarction and stroke: a new preventive strategy. Health Technol Assess 2003; 7: 1-94.

16. Mancia G, Saino A, Grassi G. Interactions between the sympathetic nervous system and rennin angiotensin system, Hypertension: Pathophy- siology, Diagnosis and Management. New York: Raven Press, 1995; p 399-407.

17. Hamada T, Watanabe M, Kenda T et al. Evaluation of changes in sympathetic nerve activity and heart rate in essential hypertensive patients induced by amlodipine and nifidipine. J Hypertens. 1998; 16 : 111-18.

18. Ylitalo A, Airaksinen J Sellin L, Huikuri HV. Effect of combination antihypertensive therapy on baroreflex sensitivity and heart rate variability in systemic hypertension. Am J cardiol. 1999; 83: 885-889.

19. Baguet JP, Fagret D, Tremel F, Gaudemaris R, Mallion J. Effects of amlodipine on baroreflex and sympathetic nervous system activity in mild to moderate hypertension. Am. J Hypertens. 2001; 14: 424-428.

20. Chern CM, Hsu Hy. Hu HH, chen YY, Hsu LC and Chao AC. Effect of atenolol and Losartan on Baroreflex Sensitivity and Heart Rate Variability in Uncomplicated essential hypertension. J cardiovas pharmacol TM. 2006; 47(2) :169-174.

21. Task Force Of The European Society Of Cardiology And The North American Society Of Pacing And Electrophysiology, Heart Rate Variability. Standards Of Measurement, Physiological Interpretation And Clinical Use. Circulation. 1996; 93: 1043- 65.

22. Pagani M, Lucini D. Autonomic dysregulation in essential hypertension: Insight from heart rate and arterial pressure variability. Auton. Neurosci. 2001; 90:76-82.

23. Ozdemir M, Arslan U, Turkoglu S, balecbgut S, Cengel A. Losartan improves heart rate variability and heart rate turbulence in heart failure due to ischaemic cardiomyopathy. Journal of heart failure. 2008; 13(10) : 812-7.

24. Tuininga YS. Harry JGM, Crijns HJGM, Brouwer J, Berg MPV, Veld AJMV, Mulder G, Lie KI. Evaluation of importance of central effects of atenolol and metoprolol measured by heart rate variability during mental performance tasks, physical exercise, and daily life in postinfarct patients. Circulation. 1995; 92: 3415-3423.

25. Carpia A, Antonomic F, Ciamei A, Roncoroni V, Sabino D, Fontana L. Short-term effects of Losartan on autonomic nervous system. Am. J. Hypertension. 2000; 13(52): 149A.

26. Sahin I, Kosar F, Altunkan S, Gunaydin M.Comparison of the effects of amlodipine and 
verapamil on autonomic activity in hypertensive patients. Eur J of intern med (2004) ; 15: 225-230.

27. Rabbia F, Silke B, Carra R, Milan A, Colle SD, Pugni C, Mulatero P, Chiandussi L. Heart rate variability and Baroreflex Sensitivity during fosinopril, Irbesartan and atenolol therapy in hypertension. Clin Drug Invest. 2004; 24(11).

28. Bilge AK, Atilgan D, Tukek T, Ozcan M, Ozben B, Kuylan B, Meric M. Effects of amlodipine and fosinopril on heart rate variability and left ventricular mass in mild-to-moderate essential hypertension. Int J clin pract. 2005; 59(3): 30610.

29. Karas M, Lacourciere Y, Le Blanc RA, Nadeau R, Dube B, Florescu M et al. Effect of renninangiotensin system or calcium channel blockade on circadian variation of heart rate variability, blood pressure and circulating catecholamines in hypertensive patients. J of Hypertens. 2005; 23 :1251-1260.

30. Pavithran P, Prakash ES, Dutta TK. Madanmohon T. Effect of antihypertensive drug therapy on short term heart rate variability in newly diagnosed essential hypertension. Clin. Exp. Pharmacol. Physiol. 2010;37 : e107-e113.
31. Colledge NR, Walker BR and Ralston SH. Davidson's Principles and Practice of Medicine. $21^{\text {st }}$ ed. China: Elsevier Limited.2010.

32. Zaliunas R, Brazdzionyte J, Zabiela V, Jurkevicius R. Effects of amlodipine and lacidipine on heart rate variability in hypertensive patients with stable angina pectoris and isolated left ventricular diastolic dysfunction. Int J Cardiol 2005; 101:347-35.

33. Chowdhury S, Townend JN. Nitric oxide and hypertension: not just an endothelium derived relaxing factor. J Hum Hypertension 2001; 15: 219-227.

34. Barrett CJ, Ramchandra R, Guil SJ, Lala A, Budgett DM, Malpas SC. What sets the long term level of renal sympathetic nerve activity? Circ. Res. 2001; $92: 1330-6$

34. Chowdhury S, Vaile JC. Fletcher J, et al. Nitric oxide and cardiac autonomic control in human. Hypertension. 2000; 36: 264-269

35. Kailasam MT, Parmer RJ, Cervenka JH, Wu RA, Ziegler MG, Kennedy BP, Adegbile IA, O’Connor DT. Divergent effects of dihydropyridine and phenylalkylamine calcium channel antagonist classes on autonomic function in human hypertension. Hypertension. 1995; 26(1) : 143-149. 